\title{
RHIC IR QUADRUPOLES AND FIELD QUALITY STATE OF THE ART IN SUPERCONDUCTING ACCELERATOR MAGNETS*
}

\author{
R. Gupta", Lawrence Berkeley National Laboratory, Berkeley, CA 94720, USA, \\ M. Anerella, J. Cozzolino, A. Ghosh, A. Jain, S. Kahn, E. Kelley, G. Morgan, \\ J. Muratore, A. Prodell, W. Sampson, P. Thompson, P. Wanderer, E. Willen, \\ Brookhaven National Laboratory, Upton, NY 11973, USA
}

\begin{abstract}
The interaction region (IR) quadrupoles [1] for the Relativistic Heavy Ion Collider (RHIC) [2] are the best field quality superconducting magnets ever built for any major accelerator. This field quality is primarily achieved with the help of eight tuning shims [3] that remove the residual errors from a magnet after it is built and tested. These shims overcome the limitations from the typical tolerances in parts and manufacturing. This paper describes the tuning shims and discusses the evolution of a flexible approach that allowed changes in the design parameters and facilitated using parts with significant dimensional variations while controlling cost and maintaining schedule and field quality. The RHIC magnet program also discovered that quench and thermal cycles cause small changes [4] in magnet geometry. The ultimate field quality performance is now understood to be determined by these changes rather than the manufacturing tolerances or the measurement errors.
\end{abstract}

\section{INTRODUCTION}

To increase the design luminosity of RHIC, the design beam size was made smaller at the crossing point [5]. An unavoidable consequence was an increase in the good field aperture required in the interaction region quadrupoles. A magnet aperture larger than the design $130 \mathrm{~mm}$ would have caused delay and increase in cost. A tuning shim method that extended the good field region was developed. As compared to the $80 \mathrm{~mm}$ aperture main arc quadrupoles, the increase in beam size in these magnets is $\sim 400 \%$, whereas the increase in aperture is only $63 \%$. In the high luminosity lattice configuration, the field errors in these magnets are more critical than those in the rest of the RHIC machine magnets combined [5]. The skew $\left(a_{n}\right)$ and normal $\left(b_{n}\right)$ field harmonics are defined (in units) as follows:

$$
B_{y}+i B_{x}=10^{-4} G \times R \sum_{n=0}^{\infty}\left[b_{n}+i a_{n}\right]\left[\frac{x+i y}{R}\right]^{n} \text {, }
$$

\footnotetext{
* Work supported by the U.S. Dept. of Energy under contract numbers DE-AC02-98CH10886 and DE-AC03-76SF00098. 'Email: RGupta@lbl.gov
}

where $\mathrm{B}_{\mathrm{x}}$ and $\mathrm{B}_{\mathrm{y}}$ are the components of the field at $(\mathrm{x}, \mathrm{y})$, $\mathrm{G}$ is the gradient at the center of the quadrupole and $R$ is the reference radius, which is $40 \mathrm{~mm}$ in these magnets.

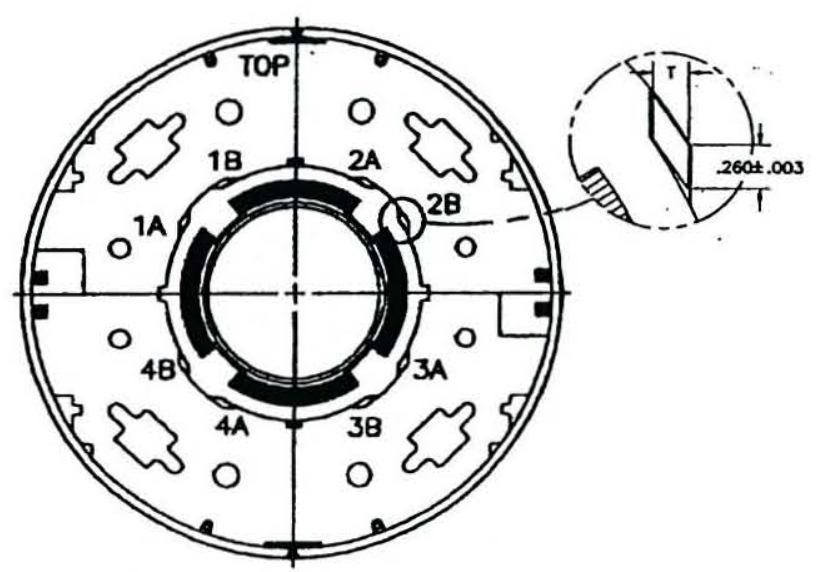

Fig. 1. Cross section of the $130 \mathrm{~mm}$ aperture RHIC interaction region quadrupoles. $1 \mathrm{~A}, 1 \mathrm{~B}$, etc. are the locations of eight gaps for tuning shims. The tuning shim area at location $2 \mathrm{~B}$ is shown in more detail.

\section{MAGNET CROSS SECTION}

The cross section of the $130 \mathrm{~mm}$ aperture insertion quadrupole is shown in Fig.1. In this design the yoke acts as a collar, a cost saving feature which is common to most RHIC magnets. An identical cross-section is used for 24 each of quadrupoles Q1 $(1.44 \mathrm{~m}), \mathrm{Q} 2(3.4 \mathrm{~m})$ and Q3 $(2.1 \mathrm{~m})$. The parameters of these magnets are given in an earlier paper [1]. A 2-radius aperture yoke reduces the computed saturation-induced $b_{5}$ from about 17 units to less than 1 unit up to $8 \mathrm{kA}$. The design current is $5 \mathrm{kA}$ and measurements performed to $\sim 7 \mathrm{kA}$ agree with these calculations. The tuning shims are inserted at eight angular locations (see Fig. 1) between the yoke and the RX630 spacers.

Another cost saving feature of all RHIC quadrupoles is the use of a 2-fold symmetric yoke design and collaring operation. This simplifies the magnet construction but breaks the 4-fold quadrupole symmetry. A large ( 7 units) measured octupole $\left(b_{3}\right)$ harmonic in the first 
assembly was compensated by deliberately introducing an asymmetry in the coil to coil ground plane insulation (midplane gap) in later designs. This, however, generated a higher order $b_{7}$ harmonic. A proper choice of asymmetric tuning shim configuration and asymmetric midplane gaps removed both $b_{3}$ and $b_{7}$ harmonics.

\section{A FLEXIBLE MAGNET PROGRAM}

In a program where only a small number of magnets are required, a significant cost is associated with the magnet R\&D to achieve the required quench and field quality performance. A large field margin, a flexible approach and an inherently inexpensive design were the primary factors in keeping the program cost low while producing high field quality for these insertion magnets.

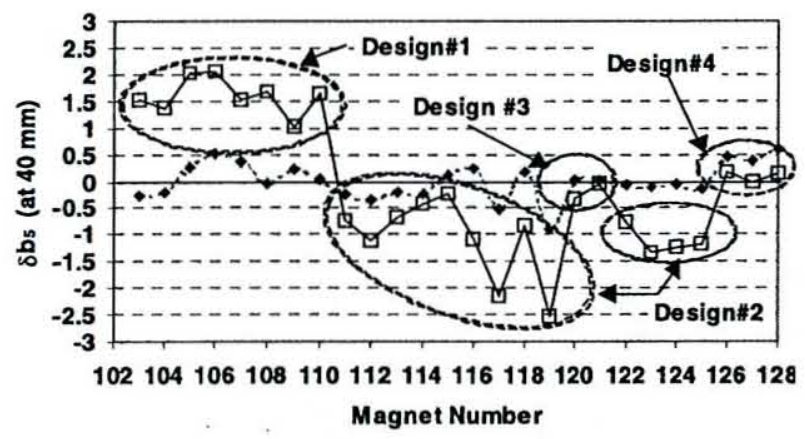

廿-b5 Errors before Tuning Shims ....

Fig. 2. A flexible design accommodated large changes in harmonics and parts without iterating the coil cross section in Q1 magnets. The figure shows the $b_{5}$ error from nominal value before (warm measurements) and after tuning shims (cold measurements at $5 \mathrm{kA}$ ).

At the time when this quadrupole cross section was being designed, the choice for cable insulation could not be made between two that would make the effective cable thickness differ by about 22 micron ( 0.9 mil). This is about an order magnitude larger than that required before a typical magnetic design can be specified. This problem was solved by choosing a unique cross section in which such a large difference (620 micron over 27 turns) was absorbed in a rectangular wedge while maintaining a constant $b_{5}$ and pole angle. Since the pole angle did not change, the coil curing tooling and the spacer dimensions could be specified and the magnet design and construction were not delayed.

In Q1 magnets, the midplane gaps were adjusted between 150 micron and 390 micron (rather than a fixed typical value of 100 micron) and pole shims between 430 micron and 630 micron. This flexibility was used in a combination that kept the pre-stress on the coil within the desired range, while adjusting the field harmonics and accommodating variation in parts. As shown in figure 2, a large change in $b_{5}$ in design\#2 and onward (required from beam dynamics considerations), a mid- course correction for drift in $b_{5}$ (design\#4), and a large 9 micron change in cable size or 243 micron in coil size (design\#3), were accommodated. As mentioned in the last section, a large $b_{3}$ harmonic was also removed by adjusting midplane gaps. In the past, such changes required an iteration in the coil cross section, causing delay in production and increase in cost.

\section{TUNING SHIMS}

Eight tuning shims of adjustable iron thickness to adjust eight harmonics $\left(a_{2}\right.$ through $a_{5}$ and $b_{2}$ through $\left.b_{5}\right)$ are used in each magnet. The nominal resolution of the harmonic correction, determined by the minimum thickness of iron lamination $(0.125 \mathrm{~mm})$ in the tuning shim package, is given in Table 1 . The resolution in certain harmonics is better for certain location of tuning shim, but that may not always be realized when eight harmonics are optimized together. The resolution given in Table 1 is generally comparable to the uncertainties in the measured harmonics within a factor of 2 . The reproducibility of the harmonic measurements itself was, however, an order of magnitude better than that listed in Table 1. The maximum thickness of iron in each tuning shim package is $6 \mathrm{~mm}$.

Table 1. Resolution in the harmonic correction from the tuning shim package at the design current.

\begin{tabular}{|c|c|c|c|c|c|c|c|}
\hline $\mathbf{a}_{2}$ & $\mathbf{a}_{3}$ & $\mathbf{a}_{4}$ & $\mathbf{a}_{5}$ & $\mathbf{b}_{2}$ & $\mathbf{b}_{3}$ & $\mathbf{b}_{4}$ & $\mathbf{b}_{5}$ \\
\hline 0.2 & 0.1 & 0.07 & 0.03 & 0.2 & 0.08 & 0.07 & 0.02 \\
\hline
\end{tabular}

The iron fraction for eight tuning shims is computed to minimize the harmonics at the maximum design current (nominal value $5 \mathrm{kA}$ ) based on warm magnetic measurements. The tuning shim specifications required a series of tasks to be performed in a synchronous and iterative way. These tasks include: (a) an error function minimization using the weighted contributions of eight primary and eight higher order harmonics, (b) a series of non-linear iron calculations (which automatically generated input for a finite element mesh program), (c) transferring data between a number of computers, (d) generating an electronic spec-sheet for manufacturing tuning shims, and (e) computing the expected warm and $5 \mathrm{kA}$ harmonics after installation of tuning shims.

Each tuning shim is actually a package of fifteen (four $0.125 \mathrm{~mm}$ thick and eleven $0.5 \mathrm{~mm}$ thick) laminations iron (magnetic) and brass (non-magnetic) - having a width of $6.6 \mathrm{~mm}$. In the initial magnets, the laminations were bonded with epoxy that was found to delaminate on cool down. In the later magnets, the laminations were simply welded in the front end. The full-length tuning shims are inserted through eight holes in one end of the magnet. The magnetic forces in the iron assure a proper contact for the tuning shim iron inside the magnet once the magnet is energized. 
The tuning shims significantly reduced $b_{5}$ errors in Q1 magnets (see Fig. 1). Table 2 gives the systematic and standard deviations from nominal value in normal harmonics $\left(b_{n}\right)$ before and after tuning shims in all magnets. A significant improvement can be seen in harmonics $b_{2}$ through $b_{5}$. However, as a result, the higher order harmonics $\left(b_{6}\right.$ through $\left.b_{9}\right)$ get worse, as expected, but remain of the order of 0.1 unit. A similar effect has been seen in skew harmonics also. Fig. 3 shows the standard deviations of field errors from nominal skew harmonics before and after tuning shims.

Table 2. The errors from the nominal values of the normal harmonics before and after tuning shims.

\begin{tabular}{|c|c|c|c|c|c|c|}
\cline { 2 - 7 } \multicolumn{1}{c|}{} & \multicolumn{2}{c|}{$\left\langle\mathbf{b}_{\mathbf{n}}\right\rangle(\mathrm{n}=2$ is sextupole) } & \multicolumn{3}{c|}{$\sigma\left(\mathbf{b}_{n}\right)$} \\
\hline $\mathbf{n}$ & NO-shim(W) & Shimsi(W) & Shims(5kA) & NO-shim(W) & Shims(W) & Shlms(5kA) \\
\hline 2 & 0.41 & 0.01 & 0.05 & 1.74 & 0.41 & 0.56 \\
\hline 3 & 0.87 & -0.76 & 0.08 & 1.19 & 0.60 & 0.49 \\
\hline 4 & 0.06 & 0.03 & -0.17 & 0.42 & 0.20 & 0.27 \\
\hline 5 & -0.07 & 0.00 & 0.05 & 0.78 & 0.78 & 0.36 \\
\hline 6 & 0.01 & 0.05 & 0.05 & 0.11 & 0.21 & 0.18 \\
\hline 7 & -0.26 & -0.07 & -0.14 & 0.04 & 0.17 & 0.14 \\
\hline 8 & 0.00 & 0.01 & 0.04 & 0.03 & 0.04 & 0.06 \\
\hline 2 & -0.03 & -0.30 & -0.14 & 0.17 & 0.18 & 0.19 \\
\hline
\end{tabular}

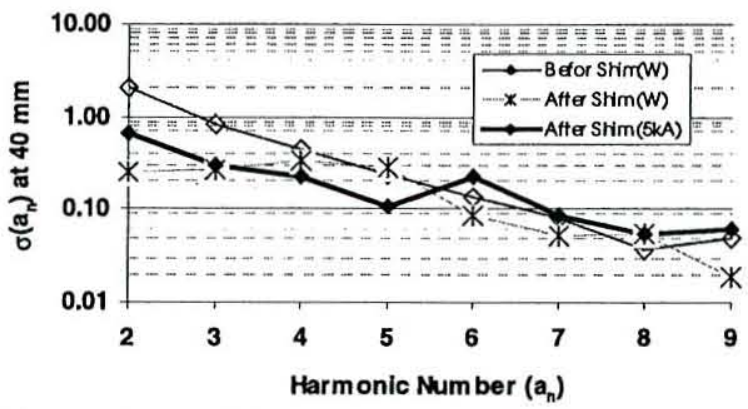

Fig. 3. Measured improvements in the Std. deviations of the lower order skew harmonics with tuning shims.

\section{DYNAMIC CHANGES}

The RHIC magnet program found that the field harmonics change after quench or thermal cycles [4]. Though these changes are relatively small, they are an important limitation in these high field quality magnets. An estimate of the magnitude of these changes (based on limited measurements) is given in Table 3 . For nonallowed harmonics the variation is of a random nature. For the allowed $b_{5}$ harmonic and to some extent also for the semi-allowed $b_{3}$ harmonic, the change is also systematic and seems to approach an asymptotic value.

Table 3. Standard deviation $(\sigma)$ of the changes in harmonics due to quench and thermal cycle in RHIC 130 $\mathrm{mm}$ aperture quadrupoles. $\sigma$ of warm-cold (W-C) correlation is also given.

\begin{tabular}{|c|c|c|c|c|c|c|c|c|}
\hline & $\mathbf{a}_{\mathbf{2}}$ & $\mathbf{a}_{3}$ & $\mathbf{a}_{4}$ & $\mathbf{a}_{5}$ & $\mathbf{b}_{\mathbf{2}}$ & $\mathbf{b}_{3}$ & $\mathbf{b}_{4}$ & $\mathbf{b}_{5}$ \\
\hline Quench & 0.4 & 0.3 & 0.1 & 0.01 & 0.4 & 0.1 & 0.1 & 0.1 \\
\hline Thermal & 0.3 & 0.1 & 0.1 & 0.01 & 0.3 & 0.1 & 0.05 & 0.1 \\
\hline W-C & 0.7 & 0.3 & 0.2 & 0.1 & 0.5 & 0.4 & 0.25 & 0.2 \\
\hline
\end{tabular}

Similar changes have been observed in other RHIC magnets, in SSC magnets (but having smaller magnitude) and recently in 5-block LHC dipoles [6].

\section{ULTIMATE FIELD QUALITY}

Although the field errors in these magnets are significantly smaller than ever measured before, there is still a room for improvement. The tuning shim resolution (see Table 1) did not limit the performance and the harmonic measurement system has made large progress over time [7]. One source of remaining errors was the uncertainty due to warm-cold correlation as the tuning shim correction was based on estimating cold harmonics from the warm measurements. "W-C" in Table 6 includes the errors because of different measuring coils and a different setup of the lead configuration in two cases. In principle, this error could have been reduced, if desired, by applying harmonic correction to the cold measurements and measuring harmonics in a setup similar to that in the machine. This would, however, have improved the situation only moderately. The ultimate limit is now placed by the changes due to quench and thermal cycles, as discussed in the last section. To fully utilize the capability of the tuning shim method in future magnets, these changes must be studied systematically so that they can be made smaller. It appears that the magnitude of these changes depends on the details of magnet construction.

Sorting [8] allowed a better field quality in the critical magnets as only 2 out of 6 interaction regions will be operated in the high luminosity configuration.

\section{ACKNOWLEDGEMENTS}

The above developments would not have been possible without close interaction between the RHIC accelerator physicists and magnet scientists. In particular, discussions with Jie Wei and Steve Peggs and encouragements from Mike Harrison were critical to this development. The role of technicians is also appreciated.

\section{REFERENCES}

[1] R. Gupta, et al., "Large Aperture Quadrupoles for RHIC Interaction Region", Proc. of the 1993 Particle Accl. Conference, Washington D.C., pp 2745-7.

[2] M. Harrison, "RHIC Status", these proceedings.

[3] R. Gupta, et al., "Tuning Shims for High Field Quality in Superconducting Magnets", Proc. of MT-14, IEEE Trans. on Magnetics, Vol. 32, No. 4, 1996, pp. 2069-73.

[4] R. Gupta, et al., "Changes in Field Harmonics after Quench and Thermal Cycles in Superconducting Magnets", Proc. of the 1997 Particle Accl. Conference, Vancouver, Canada, pp 3347-9.

[5] J. Wei, et al., "Magnetic Correction for RHIC Triplets", Proc. of the 1993 Particle Accl. Conference, Washington D.C., pp 258-260.

[6] W. Scandale, et al., "Field-Shape Variation Induced by Thermal Cycling and Excitation in the LHC Dipole", these proceedings.

[7] A. Jain and P. Wanderer, BNL note RHIC-MD-281, Jan 25, 1999.

[8] J. Wei, et al., "Real World Sorting of Superconducting Magnets in the Relativistic Heavy Ion Collider", these proceedings. 